\title{
Modelling and Prediction of Precipitation and Soil Movement Based on ADInSAR
}

\author{
C. H. Yang *, A. Müterthies \\ EFTAS Remote Sensing Transfer of Technology, Münster, Germany - (chia-hsiang.yang, andreas.mueterthies)@eftas.com
}

KEY WORDS: Precipitation, Soil Movement, SAR Interferometry, Advanced DInSAR, Sentinel-1

\begin{abstract}
:
Evaluating soil movement related to precipitation is needed for geologic and hydrologic applications. In principle, the soil body swells and shrinks depending on soil type, precipitation rate, moisture content, and drainage rate. The precipitations are normally measured at weather stations. Measuring the soil movement by using ground-based sensors and hydrologic models across a large area is costly and time-consuming. Also the weather observations were not fully involved in modelling. A long-term monitoring using remote sensing is a cost-effective alternative. For this purpose, we developed a new approach in this study to model the transformation between precipitation and soil movement. The time-series soil movement over a large area is evaluated by ADInSAR at mm/yr level. As a result, the predictive model can compute the precipitation at a location from its ADInSAR-derived movement, and vice versa. Our test using Sentinel-1 images shows that the prediction accuracy for precipitation is $14 \mathrm{~mm}$ (mean error rate $12 \%$ ) and it amounts $12 \mathrm{~mm} / \mathrm{yr}$ for soil movement. The accuracies indicate that our modelling is relevant to the reality. We also discuss the influences of different parameters on the modelling. In the future, we will proceed with tests considering other areas of interest, time spans, and image sources. More target points will be analysed in detail. Last but not least, we will work on the applications related to geology and hydrology.
\end{abstract}

\section{INTRODUCTION}

Evaluating soil movement is needed for geologic and hydrologic applications, e.g., to understand ecological essence of moor. In principle, when the water infiltrates via, e.g., rainfall, and stay under the soil surface, the soil swell leads to heave movement. In contrast, subsidence occurs resulting from soil shrink as the moisture dries out. To measure this movement by using groundbased sensors and hydrologic models across an extensive area is not cost-effective.

Differential interferometric SAR (DInSAR) using a pair of spaceborne SAR images is an alternative to detect soil movement over a large area (Barrett et al., 2013). The approach evaluates the soil movement between two image acquisitions up to $\mathrm{mm}$ level. The other kinds of movement are usually excluded by assumption. Some previous works focused on exploring and modelling the transformation between soil moistures and interferometric values like phase and coherence (De Zan et al., 2015; Zwieback et al., 2015a, 2015b, 2017). Their goal is to separate the component subject to soil moisture from the actual movement of interest such as mining-induced subsidence. One limitation is that only the soil movement between two image acquisitions is evaluated each time. So far a time-series monitoring was barely mentioned. Also the weather observations were not fully involved in modelling.

In this study, we explore the mechanism between soil moisture and its movement (Grant and Aitchison, 1970). The observations associated with soil moisture are precipitations, which are hourly measured at weather stations of Deutscher Wetterdienst (DWD). The movement is evaluated via advanced DInSAR (ADInSAR) (Berardino et al., 2002; Ferretti et al., 2000, 2001, 2011; Lanari et al., 2004, 2007). By using multi-temporal SAR images, ADInSAR evaluates the long-term movement over an extensive area up to submillimetre level. We then model the transformation between precipitation and soil movement. As a result, the predictive model can compute the precipitation at a location from its ADInSAR-derived movement, and vice versa.

Our approach contributes to new applications for geology, hydrology and SAR interferometry. For geology and hydrology, although weather stations provide accurate precipitation measurements, they cannot be unlimitedly distributed in practice considering cost and logistic. Normally, an interpolation is applied to generate large-scale precipitation data. A comparable precipitation dataset derived from the soil movement can be combined to improve the accuracy. The improvement is effective particularly for extrapolated precipitations. One important use for SAR interferometry is to separate soil movement from actual movement. A predictive model is first created from a reference site, where the soil movement only regards moisture content. This model utilizes precipitations at the area of interest to compute the local moisture-induced movement. This component is then subtracted from the ADInSAR-derived movement to obtain the actual movement, if any.

In this paper, we first describe our method in Section 2. Section 3 illustrates our experiments at a moor area in Germany. We demonstrate how to generate a predictive model and evaluate the accuracy by using real data. The influences of different parameters on the modelling are statistically analysed. Finally, this study is concluded in Section 4.

\section{METHODOLOGY}

\subsection{Mechanism of soil movement}

In view of geology and hydrology, soil movement is triggered and influenced by temperature and precipitation (Figure 1). When the precipitation infiltrates the soil, the moisture content is increased, which makes the soil body swell. Conversely, the soil body shrinks after the moisture content evaporates and drains. The evaporation is accelerated and decelerated as the temperature

\footnotetext{
* Corresponding author
} 
rises and drops, respectively. The drainage speed and mass are subject to geologic characteristics. The interaction between swell and shrink of a soil body results in soil movement. This movement can be detected from the surface via SAR interferometry such as ADInSAR.

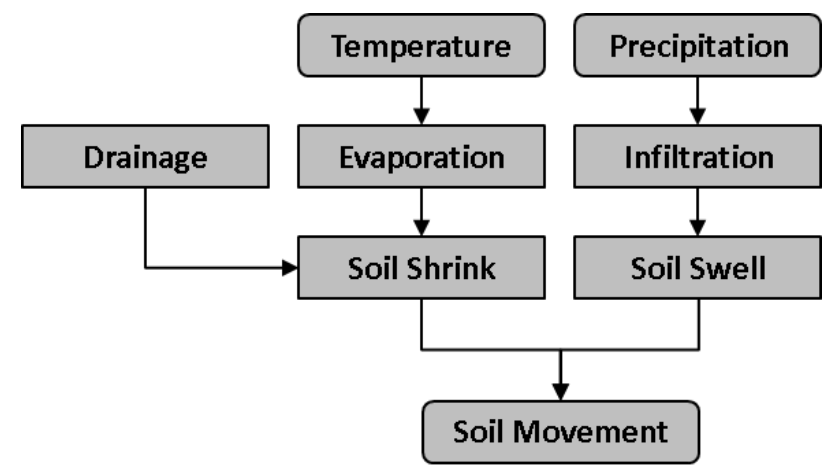

Figure 1. Mechanism of soil movement influenced by geologic and hydrologic factors.

\subsection{ADInSAR}

ADInSAR operates as a long-term monitoring over a large-scale area up to submillimetre level. This technique processes a time series of SAR images to detect target points of interest. Each target point corresponds to a ground patch of a certain size depending on the image resolution. The signals are reflected back to the SAR antenna under a repeat cycle. Those signals characterized by high coherence are used to evaluate the local movements of different forms, e.g., cumulative time series or average velocity.

In this study, we use the small baseline subset (SBAS) method (Berardino et al., 2002; Lanari et al., 2004, 2007, 2010; Pepe et al., 2015; Tizzani et al., 2007) to compute soil movement. From a SAR image set, SBAS generates interferograms characterized by limited spatial and temporal baselines. The so-called small baseline constraint mitigates scene coherence loss caused by temporal and geometric decorrelations. This advantage favours detection of target points on bare soil as well as the resultant movement precision.

The basic SBAS processing is explained in the following. The interferogram sets are temporally connected by singular value decomposition (Golub and Van Loan, 1996). A pixel is selected as a target point if its ensemble coherence of interferograms fulfils a specified threshold. Afterwards, all of the target points are spatially connected by Delaunay triangulation to form a network of target points. The interferometric phases of the target points are unwrapped (Costantini and Rosen, 1999) and then used to interpolate the phases of the other non-target points. A transformation is modelled considering the unwrapped interferometric phases and the corresponding movements. The movements are evaluated via a least square algorithm (Teunissen, 2000). The evaluation accuracy is further improved by iteration after removal of atmospheric phase screen (APS). For this purpose, the APS-like phases are derived by means of a low-pass spatial filtering and a high-pass temporal filtering.

The selection of a coherence threshold leads to a trade-off between quality and quantity of target points. A complex coherence is commonly expressed as (Bamler and Hartl, 1998)

$$
\gamma=\frac{\mathrm{E}\left[s_{\mathrm{t} 1} \cdot s_{\mathrm{t} 2}^{*}\right]}{\sqrt{\mathrm{E}\left[\left|s_{\mathrm{t} 1}\right|^{2}\right] \cdot \mathrm{E}\left[\left|s_{\mathrm{t} 2}\right|^{2}\right]}}
$$

where $s_{\mathrm{t} 1}$ and $s_{\mathrm{t} 2}$ are complex radar signals acquired at different times. The magnitude $|\gamma|[0,1] \in \mathrm{R}$ is customarily called coherence. In SBAS, the coherence of each point is extended to be an ensemble coherence, which is evaluated from all temporally-connected SAR images (Lanari et al., 2004). Given a high threshold, we anticipate a precise movement estimation while the number of target points is restrained. In case of a strict threshold, the clusters of sparse target points might not sufficiently cover the areas of interest. Simply speaking, there is no standard answer to an optimal threshold. We must consider actual situations to make an adequate decision. In our monitoring works, we will derive movement precisions from coherences as

$$
\sigma_{\mathrm{m}}=\frac{\lambda}{4 \pi} \sqrt{\frac{1-|\gamma|^{2}}{2 \cdot|\gamma|^{2}}}
$$

where $\lambda$ means wavelength. The unit applies to the metric, e.g., millimetre. This movement precision can be converted into the velocity precision as

$$
\sigma_{\mathrm{v}}=\frac{\lambda}{4 \pi \cdot B_{\mathrm{T}}} \sqrt{\frac{1-|\gamma|^{2}}{2 \cdot|\gamma|^{2}}}
$$

where $B_{\mathrm{T}}$ is the time span of an image series. The metric is divided by a temporal interval, like millimetre per year.

\subsection{Modelling}

According to our experience, the influence of precipitation on soil movement prevails over temperature. This is also true due to the sensitivity limitation of the contemporary SAR sensors. We reform the mechanism of soil movement (Figure 1) into the new one (Figure 2) considering only precipitation. We have validated this assumption in our experiments (Section 3).

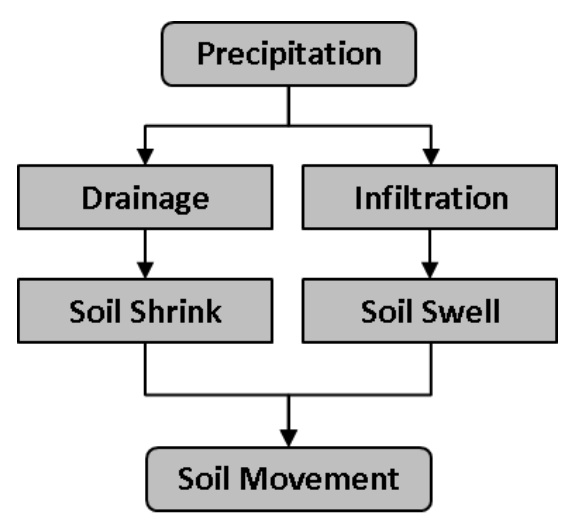

Figure 2. Influence of precipitation on soil movement.

The mechanism between precipitation and soil movement is assumed as follows. The precipitations infiltrate the soil surface and are retained in the soil body. The more moisture content, the larger the soil body swells. In contrast, the soil body shrinks due to drainage. The influences of soil type, precipitation rate, moisture content, and drainage rate on the soil movement are extended, progressive, and overlapping. Usually, they cannot be accurately defined. For example, a downpour lasting half an hour might not cause any detectable movement as the moisture content might drain quickly. In case of a heavy rainy season, the area heaves acceleratively until the rain eases. 
Our goal is to invent a reasonable model, which can be mathematically solved in a simply way. Our observations include precipitation and soil movement. The precipitations are hourly measured at weather stations. The soil movement evaluated by SBAS is expressed as a discrete time series. In reality, the soil movement is a continuous course rather than a discrete displacement. We thus abstractly and physically convert the observations into a continuous form. The movement series is first modelled by a polynomial fitting to be a continuous form. The corresponding velocities can be derived by differentiation. Our model for a target point is expressed based on an $n$-order polynomial as

$$
V_{\mathrm{M}}=\sum_{k=0}^{n} a(k) \cdot P_{\mathrm{A}}^{k}
$$

where $V_{\mathrm{M}}$ indicates an instantaneous movement velocity, $a$ signifies coefficient, and $P_{\mathrm{A}}$ amounts an accumulated precipitation within a period. Our model signifies that the accumulated precipitation during a period causes the local movement. This local movement is expressed as the instantaneous movement velocity at the middle of this period.

The model (4) is solved by a least square algorithm (Teunissen, 2000). Afterwards, we can predict the soil movement of a target point from its precipitation, and vice versa. The key parameter is the resampling period, within which the precipitations accumulate and the corresponding velocity is set at the middle. An optimal period can be determined based on a goodness-of-fit principle. More details are referred to Section 3.5 (especially related to Figure 13). In general, a longer period would make the precipitation and velocity more linearly correlated. Thus the model-solving becomes more stable and precise. The reason is that the extended influence of the precipitation on the soil movement can be effectively reflected. Nevertheless, the downsampling also degrades the temporal resolution. One solution is to derive velocities at times of interest via interpolation or linear fitting assuming that soil ground moves smoothly. A similar operation can be applied to precipitation while the result is irrelevant in practice.

\section{EXPERIMENTS}

\subsection{Test area and data}

Currently, the civil SAR satellites are operating mainly in X-, C-, and L-Band, for example, TerraSAR-X, Sentinel-1, and PALSAR-2, respectively. We chose Sentinel-1 images for our initial test considering the following four advantages. First, the use of C-Band leads to a compromise result between $\mathrm{X}$ - and LBands. X-Band is able to measure small deformation while the results suffer from coherence lose (accuracy degradation) especially in vegetation areas. In contrast, L-Band is more robust against such a coherence loss; however, small deformation might not be detected. Mostly, C-Band satisfies the requirements for monitoring missions. Second, a standard image package contains a large area thanks to TOPSAR acquisition mode. Such a large coverage enables synchronous monitoring of areas of interest. Third, the shortest repeat cycle of 6 days empowers weekly monitoring. Last but not least, Sentinel-1 images are free of charge for both scientific and commercial purposes.

We tested our approach at a moor area in a nature reserve called Tinner Dose-Sprakeler Heide in Germany (Figure 3). The main processing of our novel development was programmed using IDL.
The moor movement is in particular sensitive to precipitation. Our precipitation data were measured hourly in 2017 at the weather station - Groß Berßen operated by DWD. The data can be downloaded from the DWD server free of charge. We used 57 Sentinel-1 images (Figure 4) in SBAS to evaluate the moor movement. These 57 images were acquired under IW mode from January 11, 2017 to December 31, 2017 along an ascending orbit. The SBAS result is calibrated to the reference point, which is fixed on a building in Klein Berßen.

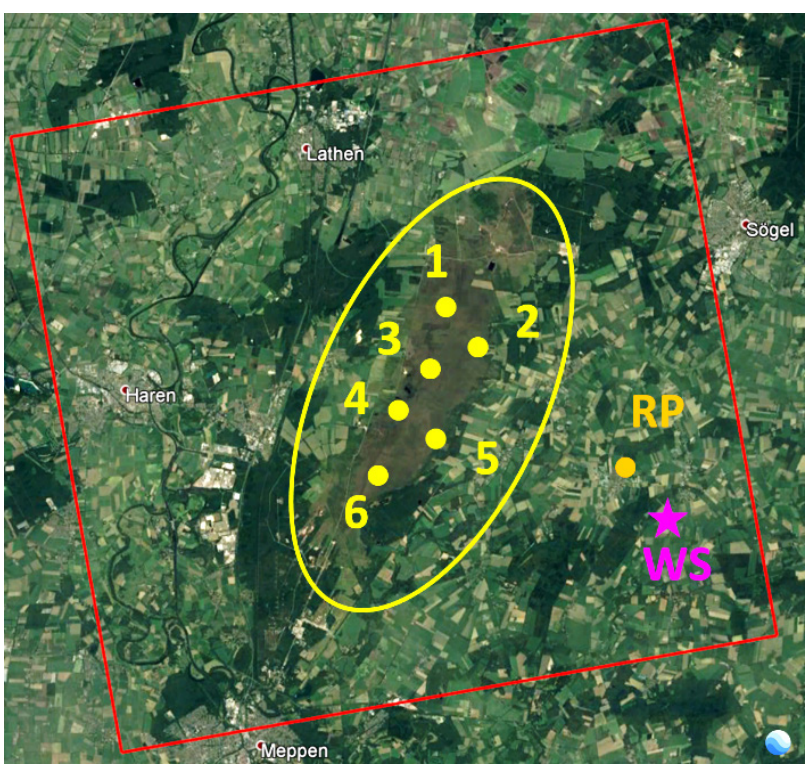

Figure 3. Test moor area (yellow circle) in nature reserve Tinner Dose-Sprakeler Heide, Germany. Target points $1-6$ used for in-depth analysis. RP, Reference Point used in SBAS. WS, Weather Station (Groß Berßen) operated by DWD. Red rectangle, coverage of Sentinel-1 images $(20 \mathrm{~km} \times 20 \mathrm{~km})$. Background downloaded from Google Earth.

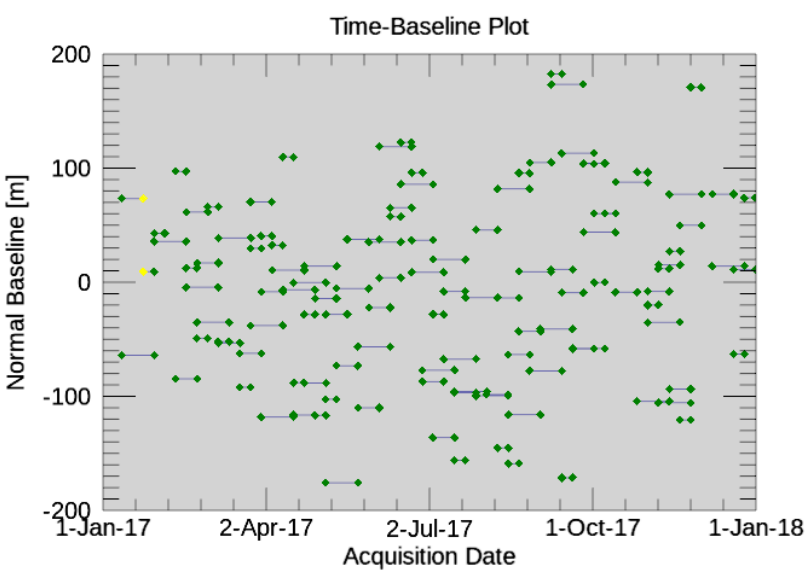

Figure 4. Normal and temporal baselines of connected image pairs in SBAS processing.

\subsection{Overview of soil movement and precipitation}

The cumulative vertical movements of the 6 target points (Figure 3 ) are evaluated via a SBAS processing and illustrated in Figure 5. The vertical movements are converted from the line-of-sight movements via Trigonometry. Before fitting, the vibration of the connected discrete movements (dots) implies the noise in the evaluations. The continuous cumulative vertical movements are generated after fitting (Figure 6). The noisy movements have been eliminated. We then derived the instantaneous vertical velocities (Figure 7) from their fitted movements. The velocity 
precision approximates $2 \mathrm{~mm} / \mathrm{yr}$. We can see that the velocities are related to the precipitations (Figure 8).

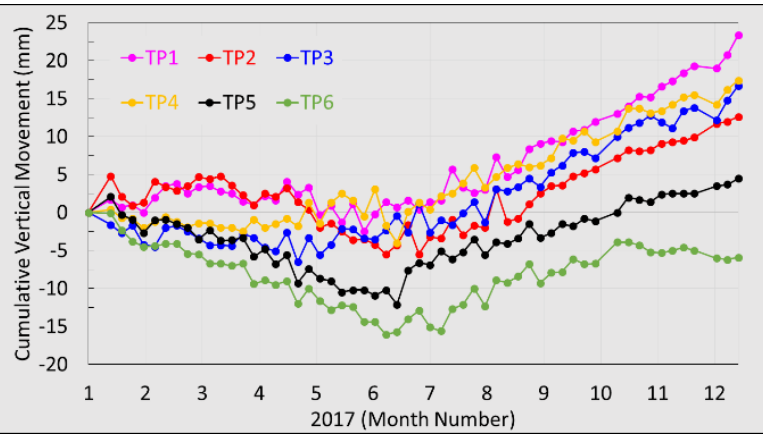

Figure 5. Cumulative vertical movement $(\mathrm{mm})$ of target points (TP) $1-6$ (Figure 3) in 2017 before fitting. Positive and negative, up and down movements, respectively.

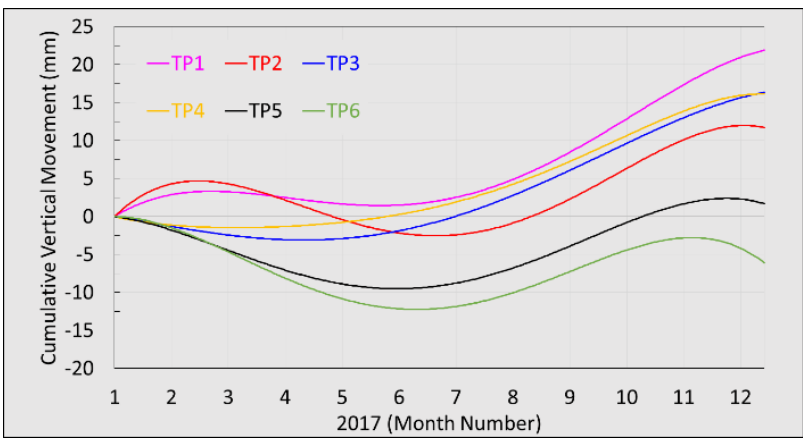

Figure 6. Cumulative vertical movement $(\mathrm{mm})$ of target points (TP) $1-6$ (Figure 3) in 2017 after fitting. Positive and negative, up and down movements, respectively.

The precipitations (Figure 8) decreased gradually from January to April. Meanwhile, the velocities (Figure 7) were tending downwards as the moisture content drained. Since May the moisture content kept a high level because plenty of precipitations continued to infiltrate. The upward velocities indicate the soil was swelling. This phenomenon was reversed roughly after October as the precipitations were not accumulated as fast as earlier. Unlike others, target point 4 was uplifting since the beginning even the precipitations reduced. We infer that the soil of target point 4 can hold its groundwater to some extent for a long time. In conclusion, the courses of the precipitations and soil movements agree with our assumption in Section 2.3.

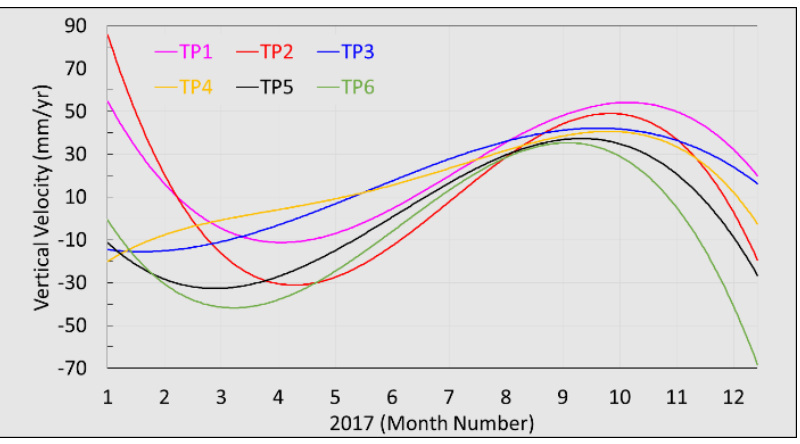

Figure 7. Vertical velocity (mm/yr) of target points (TP) $1-6$ (Figure 3 ) in 2017. Velocity precision $\approx 2 \mathrm{~mm} / \mathrm{yr}$. Positive and negative, up and down movements, respectively.

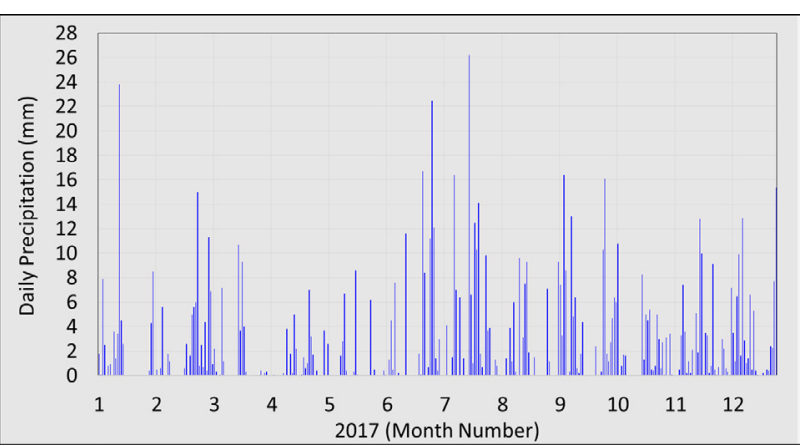

Figure 8. Daily precipitation $(\mathrm{mm})$ measured at weather station Groß Berßen (Figure 3) in 2017.

\subsection{Modelling of precipitation and soil movement}

Target point 6 was selected to demonstrate the modelling results (Figure 9). The daily precipitations (Figure 8) are resampled and accumulated every 45 days. We will discuss how to determine a resampling period in Section 3.5. Before modelling, the precipitations and SBAS velocities seem positively correlated (The Pearson correlation coefficient (PCC) is 0.95 ). The soil was moving upwards due to swell when more precipitations were accumulated. Modelling the precipitations and SBAS velocities results in a predictive model. The modelled velocities are computed from the precipitations via this model. The $\mathrm{PCC} \approx 1.00$ indicates that the precipitations and modelled velocities precisely match. We evaluate the modelling precision by comparing the SBAS and modelled velocities (Figure 10). The mean absolute difference between them amounts $10 \mathrm{~mm} / \mathrm{yr}$.

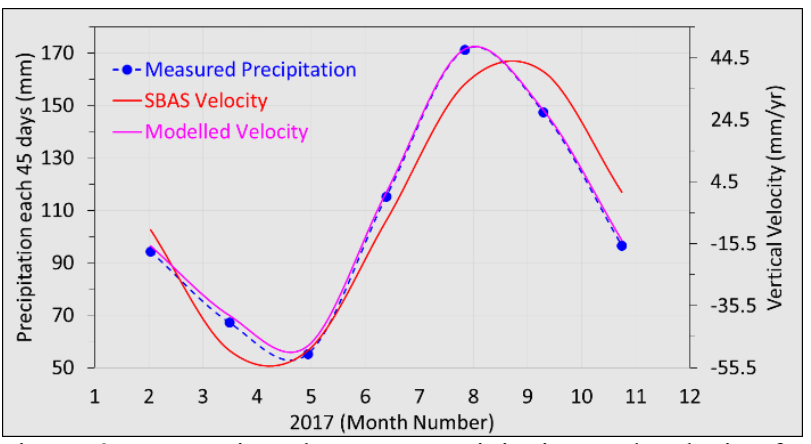

Figure 9. Comparison between precipitation and velocity for target point 6 (Figure 3). Measured precipitations are accumulated every 45 days from daily measurements (Figure 8 ).

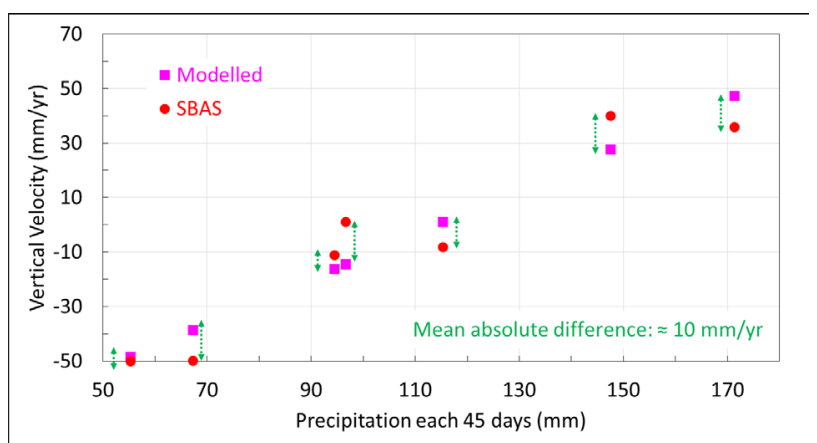

Figure 10. Comparison between SBAS and Modelled velocities for target point 6 (Figure 3).

\subsection{Predictive model}

We generated a predictive model of target point 6 (Figure 3) from the measured precipitations and the SBAS velocities in first half 
of 2017 (Figure 11). The precipitations and velocities in second half of 2017 were predicted via this model. The measured precipitations and the SBAS velocities are treated as ground truth We calculated the mean absolute difference between the predicted data and ground truth to estimate the prediction accuracy. The prediction accuracy for precipitation is $14 \mathrm{~mm}$ (mean error rate $12 \%$ ). The prediction accuracy for velocity is 12 $\mathrm{mm} / \mathrm{yr}$. The accuracies shall be acceptable considering that our modelling is a brainchild. Based on this, we believe that our modelling is relevant to the reality.

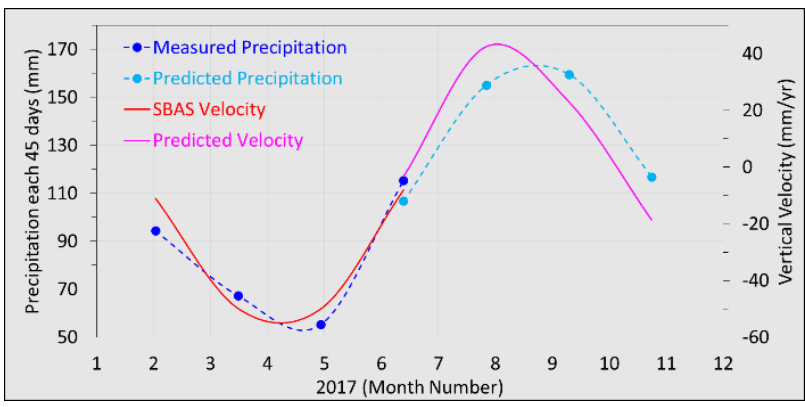

Figure 11. Predicted precipitations and velocities (second half) from measured precipitations and SBAS velocities (first half) via predictive model. Measured precipitations are accumulated every 45 days from daily measurements (Figure 8 ).

\subsection{Modelling parameters}

Our modelling approach is mainly influenced by three parameters We will discuss them in the following using target point 6 as an example. First, a cumulative movement series generated by SBAS must be converted into a continuous form via polynomial. The best-fit polynomial order is determined if its chi-square goodness-of-fit (Press et al., 1988) is smallest. For example, the $4^{\text {th }}$ order is selected in our case (Figure 12). The lower orders result in underfitting; in contrast, the higher orders lead to overfitting.

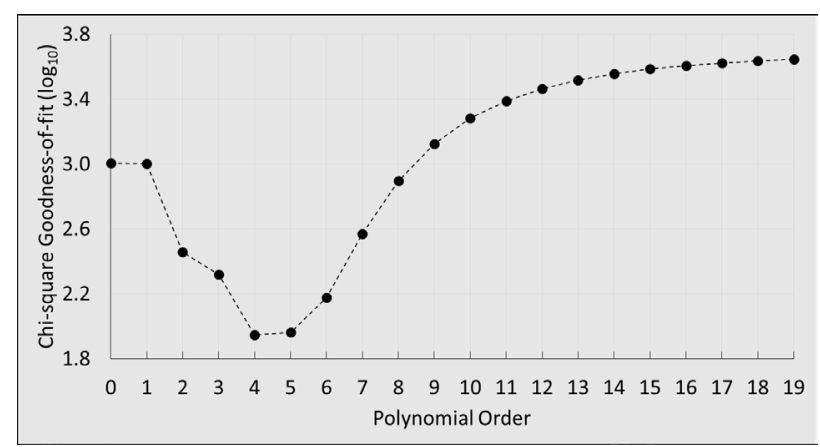

Figure 12. Chi-square goodness-of-fit regarding polynomial order to convert discrete cumulative movements (SBAS) into continuous form for target point 6 (Figure 3).

The second parameter is a resampling period, within which the precipitations accumulate and the corresponding velocity is set at the middle. As expected, the longer the period is used, the better the modelling of precipitation and velocity fits (Figure 13). Simply speaking, selecting a period is a trade-off between goodness of fit and temporal detail. We suggest to set this parameter according to the need in practice. For instance, we can evaluate the modelling accuracies of different periods by comparing ground truth, if any. Based on this, the geologists and hydrologists will find a compromise for their uses.

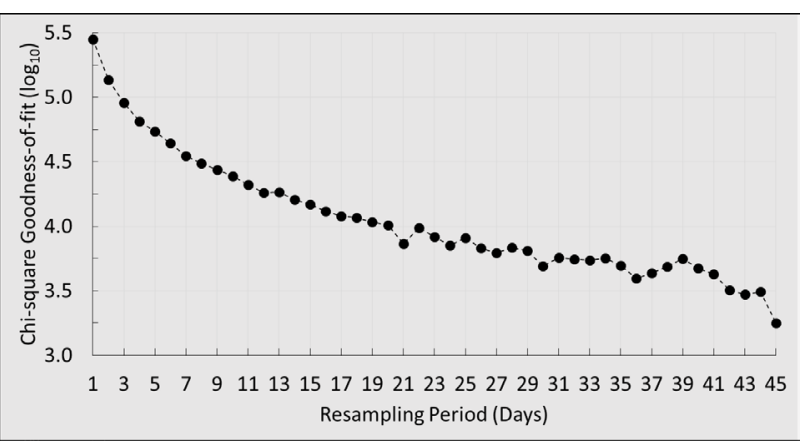

Figure 13. Chi-square goodness-of-fit regarding resampling period in modelling of precipitations and velocities for target point 6 (Figure 3 ).

The last parameter is the polynomial order of a predictive model for precipitation and soil movement. The precipitations measured at weather stations are regarded as reliable observations. We suppose that the modelled velocities are positively correlated to the measured precipitations. Based on this, the polynomial order corresponding to the highest $\mathrm{PCC}$ is used in a modelling. Our test selected the $2^{\text {nd }}$ order for target point 6 (Figure 14) as its PCC achieves $\approx 1.00$ higher than other cases. The $1^{\text {st }}$ order brings a meaningless result. The PCCs after the $2^{\text {nd }}$ order decrease gradually. We infer that the overfitting occurs after the $7^{\text {th }}$ order because the PCC begins to drop remarkably.

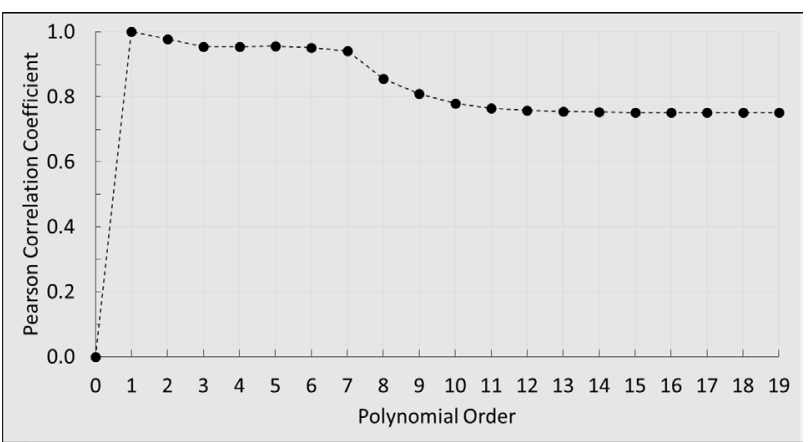

Figure 14. PCC between precipitations and modelled velocities regarding polynomial order in modelling of precipitations and velocities for target point 6 (Figure 3).

\section{CONCLUSIONS}

In this study, we proposed a new method to model precipitations and soil movements. The precipitations are measured at weather stations. The soil movements are evaluated via ADInSAR. Our modelling considers a temporal resampling to make our model close to the reality. Within each period the precipitations are accumulated and the soil movements are converted into representative velocities. These resampled data simulate that an amount of cumulated precipitations cause the soil moves progressively. A predictive model is then created based on the resampled data. Afterwards, we can predict precipitations from soil movements, and vice versa.

Our tests demonstrate reasonable and promising results. The prediction accuracy for precipitation is $14 \mathrm{~mm}$ (mean error rate $12 \%)$. The prediction accuracy for velocity is $12 \mathrm{~mm} / \mathrm{yr}$. We consider that the accuracies are acceptable for our brainchild and our modelling is relevant to the reality. Still we will consult our qualified partners about the resultant accuracies and the future validation. The influences of different modelling parameters on the modelling are also analysed and discussed. The optimal 
parameters are statistically determined based on PCC and goodness of fit. One important parameter is the resampling period, which is subject to a trade-off between goodness of fit and temporal detail. Alternatively, we should resort to acknowledged experts and set this parameter according to the need in practice.

An accurate modelling relies on accurate observations. The precipitation measurement must be reliable. We must control the precision of soil movement evaluated via ADInSAR at certain level. For future work, we will test and improve the modelling robustness and guarantee its repeatability. For this purpose, we will continue more tests regarding different areas and time spans. Meanwhile, we will investigate more point-based examples. We are currently looking for applying our technique for geology and hydrology. The current model will then be improved and adapted. Except using only precipitation, other physical factors should be also considered, such as temperature, soil type, soil roughness, electrical properties of soil, and vegetation cover.

\section{ACKNOWLEDGEMENT}

We appreciate the free accesses to the data used in this study. The Sentinel-1 images are provided by Copernicus - European Union's Earth Observation Programme. The precipitation data are obtained from Deutscher Wetterdienst.

\section{REFERENCES}

Bamler, R., Hartl, P., 1998. Synthetic aperture radar interferometry. Inverse Probl., 14(4), R1-R54.

Barrett, B., Whelan, P., Dwyer, E., 2013. Detecting changes in surface soil moisture content using differential SAR interferometry. Int. J. Remote Sens., 34(20), 7091-7112.

Berardino, P., Fornaro, G., Lanari, R., Sansosti, E., 2002. A New Algorithm for Surface Deformation Monitoring Based on Small Baseline Differential SAR Interferograms. IEEE Trans. Geosci. Remote Sens., 40(11), 2375-2383.

Costantini, M., Rosen, P.A., 1999. A Generalized Phase Unwrapping Approach for Sparse Data, in: IEEE 1999 International Geoscience and Remote Sensing Symposium. IEEE, Hamburg, Germany, pp. 267-269.

De Zan, F., Zonno, M., Lopez-Dekker, P., 2015. Phase Inconsistencies and Multiple Scattering in SAR Interferometry. IEEE Trans. Geosci. Remote Sens., 53(12), 6608-6616.

Ferretti, A., Fumagalli, A., Novali, F., Prati, C., Rocca, F., Rucci, A., 2011. A New Algorithm for Processing Interferometric Data-Stacks: SqueeSAR. IEEE Trans. Geosci. Remote Sens., 49(9), 3460-3470.

Ferretti, A., Prati, C., Rocca, F., 2000. Nonlinear Subsidence Rate Estimation Using Permanent Scatterers in Differential SAR Interferometry. IEEE Trans. Geosci. Remote Sens., 38(5), 2202-2212.

Ferretti, A., Prati, C., Rocca, F., 2001. Permanent Scatterers in SAR Interferometry. IEEE Trans. Geosci. Remote Sens., $39(1), 8-20$.

Golub, G.H., Van Loan, C.F., 1996. Matrix Computations. The Johns Hopkins University Press, Baltimore, United States

\section{of America.}

Grant, K., Aitchison, G.D., 1970. The engineering significance of silcretes and ferricretes in Australia. Eng. Geol., 4(2), 93-120.

Lanari, R., Berardino, P., Bonano, M., Casu, F., Manconi, A., Manunta, M., Manzo, M., Pepe, A., Pepe, S., Sansosti, E., Solaro, G., Tizzani, P., Zeni, G., 2010. Surface Displacements Associated with The L'Aquila $2009 \mathrm{Mw}$ 6.3 Earthquake (Central Italy): New Evidence from SBAS-DInSAR Time Series Analysis. Geophys. Res. Lett., 37(20).

Lanari, R., Casu, F., Manzo, M., Zeni, G., Berardino, P., Manunta, M., Pepe, A., 2007. An Overview of The Small Baseline Subset Algorithm: A DInSAR Technique for Surface Deformation Analysis. Pure Appl. Geophys., 164(4), 637-661.

Lanari, R., Mora, O., Manunta, M., Mallorqui, J.J., Berardino, P., Sansosti, E., 2004. A Small-baseline Approach for Investigating Deformations on Full-resolution Differential SAR Interferograms. IEEE Trans. Geosci. Remote Sens., 42(7), 1377-1386.

Pepe, A., Yang, Y., Manzo, M., Lanari, R., 2015. Improved EMCF-SBAS Processing Chain Based on Advanced Techniques for The Noise-Filtering and Selection of Small Baseline Multi-Look DInSAR Interferograms. IEEE Trans. Geosci. Remote Sens., 53(8), 4394-4417.

Press, W.H., Flannery, B.P., Teukolsky, S.A., Vetterling, W.T., 1988. Numerical Recipes in C: The Art of Scientific Computing. Cambridge University Press, Cambridge, United Kingdom.

Teunissen, P.J.G., 2000. Adjustment Theory: An Introduction. VSSD, Delft, The Netherlands.

Tizzani, P., Berardino, P., Casu, F., Euillades, P., Manzo, M., Ricciardi, G.P., Zeni, G., Lanari, R., 2007. Surface Deformation of Long Valley Caldera and Mono Basin, California, Investigated with The SBAS-InSAR Approach. Remote Sens. Environ., 108(3), 277-289.

Zwieback, S., Hensley, S., Hajnsek, I., 2015a. Assessment of soil moisture effects on L-band radar interferometry. Remote Sens. Environ., 164, 77-89.

Zwieback, S., Hensley, S., Hajnsek, I., 2015b. A Polarimetric First-Order Model of Soil Moisture Effects on the DInSAR Coherence. Remote Sens., 7(6), 7571-7596.

Zwieback, S., Hensley, S., Hajnsek, I., 2017. Soil Moisture Estimation Using Differential Radar Interferometry: Toward Separating Soil Moisture and Displacements. IEEE Trans. Geosci. Remote Sens., 55(9), 5069-5083. 